\title{
ESTIMATIVA DO COEFICIENTE DE DETERMINAÇÃO GENOTÍPICA EM MAMOEIROS (Carica papaya L.) INOCULADOS COM FUNGO MICORRÍZICO ARBUSCULAR ${ }^{1}$
}

\author{
ALDO VILAR TRINDADE²; JORGE LUIZ LOYOLA DANTAS²; FLORÍCIO PINTO DE ALMEIDA ${ }^{3}$; \\ ISABEL CRISTINA SILVA MAIA ${ }^{4}$
}

\begin{abstract}
RESUMO - O trabalho objetivou determinar o coeficiente de determinação genotípica (b) do mamoeiro (Carica papaya L.) quanto à capacidade de se associar e responder ao fungo micorrízico arbuscular (FMA) Gigaspora margarita. O experimento foi instalado em casa de vegetação da Embrapa Mandioca e Fruticultura, usando solo com uma dose única de fósforo $\left(20 \mathrm{mg} \mathrm{dm}^{-3}\right)$. Os tratamentos foram representados por quatro genótipos de cada um dos grupos 'Solo' e 'Formosa', mais as variedades 'Improved Sunrise Solo Line 72/12' e 'Tainung No 1', submetidas ou não à inoculação do fungo Gigaspora margarita. Foi utilizado um delineamento de blocos casualizados, com 4 repetições. Após a coleta dos dados, estimou-se o coeficiente "b" para os seguintes caracteres: parte aérea, comprimento de raiz, altura de planta, eficiência micorrízica e colonização. Os genótipos testados responderam à inoculação do fungo MA; os genótipos do grupo Formosa apresentaram menor comprimento de raiz; a efíciência micorrízica e a colonização radicular média para os dois grupos de plantas foram similares, situando-se em $60 \%$ e $50 \%$, respectivamente; a inoculação aumentou a absorção de $P$, $\mathrm{K}$ e $\mathrm{Cu}$, sendo esse efeito maior na variedade representante de cada grupo; o coeficiente "b" demonstrou que o melhoramento de plantas pode modular a produção de parte aérea, comprimento de raízes, altura de plantas e a eficiência micorrízica do grupo Formosa; para o grupo Solo este efeito mostrou-se mais provável para colonização radicular e menos provável para produção de parte aérea, eficiência micorrízica, comprimento de raízes e altura de plantas.
\end{abstract}

Termos para indexação: eficiência micorrízica, Gigaspora margarita, comprimento de raiz, Carica papaya.

\section{ESTIMATIVE OF THE GENOTYPIC DETERMINATION COEFFICIENT IN PAPAYA (Carica papaya L.) IN RESPONSE TO INOCULATION OF ARBUSCULAR MYCORRHIZAL FUNGUS}

\begin{abstract}
An experiment was conducted at the Embrapa - Cassava and Tropical Fruit Crops with the aim of estimating the genotypic determination coefficient (b) of papaya for its capacity of associate and response to arbuscular mycorrhizal fungi (AMF). Four genotypes and one variety of both "Solo"and "Formosa" groups were tested, inoculated or not with Gigaspora margarita in a soil which received $30 \mathrm{mg} \mathrm{dm}^{-3}$ of phosphorus. Data of shoot dry weight (SDW), mycorrhizal efficiency and colonization were statistically analized to generate the coefficient. All genotypes responded significantly to AMF but plants from "Formosa" group showed lower root length; mycorrhiza efficiency and colonization were similar for both groups, but there was no correlation between these characteristics; inoculation increased $\mathrm{P}, \mathrm{K}$ and $\mathrm{Cu}$ absorption with higher values for the variety of each group. Coefficient " $\mathrm{b}$ " showed that for "Formosa" group, plant breeding may modulate shoot plant production, root length, plant heigth and mycorrhizal effeciency; for "Solo"group this effect showed to be more probable for root colonization than to mycorrhizal efficiency.
\end{abstract}

Index terms: Mycorrhizal efficiency, Gigaspora margarita, root length, Carica papaya

\section{INTRODUÇÃO}

O mamoeiro (Carica papaya L.) é uma espécie nativa da América Tropical, intensamente cultivada nas regiões climáticas favoráveis. O Brasil é o maior produtor mundial de mamão (FAO 2000 ), participando com $34,9 \%$ do volume total produzido. Visando à melhoria da produção e à qualidade da fruta, programas intensivos de melhoramento genético têm sido desenvolvidos em países como Estados Unidos (Havaí), África do Sul, Austrália, Taiwan, Índia, México e Filipinas (GIACOMETTI, 1987).

O mamoeiro é uma planta que responde acentuadamente à formação de micorrizas onde o emprego da inoculação é prática possível em função da etapa de formação de mudas. A resposta de crescimento das plantas à simbiose micorrízica depende de três componentes principais: a planta, o fungo e as condições do solo. Para se chegar a um programa de inoculação para uma determinada cultura, é necessário conhecer o seu nível de dependência à micorrização. A dependência micorrízica tem sido definida por GERDEMANN (1975) como o "grau no qual a planta é dependente da condição micorrízica para produzir seu crescimento máximo ou produção a um dado nível de fertilidade do solo". A magnitude de resposta varia entre e dentro de espécies de plantas, sendo atribuída principalmente à habilidade destas em absorver fósforo do solo (MOSSE, 1973).

\footnotetext{
1 (Trabalho 258/2000). Recebido: 24/11/2000. Aceito para publicação: 16/10/2001.

2 Pesquisador da Embrapa Mandioca e Fruticultura, Cruz das Almas, BA, Cx. Postal 007, CEP: 44380-000. Endereços eletrônicos: aldo@cnpmf.embrapa.bre loyola@cnpmf.embrapa.br

3 Pesquisador da Empresa Baiana de Desenvolvimento Agrícola, EBDA, Luiz Eduardo Magalhães, BA, 47813-000

4 Bolsista da Embrapa Mandioca e Fruticultura, Cruz das Almas, BA. CEP. 44380-000
} 
Características morfológicas, fisiológicas ou fenológicas do hospedeiro controlam a demanda e suprimento de $\mathrm{P} \mathrm{e}$, assim, o grau de micotrofismo da planta.

De acordo com a abordagem de KOIDE (1991), a micorriza pode incrementar a performance da planta pela diminuição do déficit de $\mathrm{P}$ em função do suprimento deste nutriente, que é determinado principalmente pelo comprimento de raiz e influxo de P. Quanto maior for a demanda de $\mathrm{P}$ de um determinado hospedeiro, maior será o seu déficit, conseqüentemente, maior será o beneficio micorrízico ou a dependência da planta e maior será o nível de $\mathrm{P}$ no solo, no qual a associação pode ainda ser benéfica. Assim, dentro de um determinado solo, variação entre diferentes genótipos de uma determinada espécie de planta, com relação ao déficit de $\mathrm{P}$, deve influenciar a colonização micorrízica. Mesmo em plantas muito próximas geneticamente, como em populações de meio-irmãos, pode haver diferenças (LACKIE et al., 1988). Por conseguinte, o genótipo do hospedeiro é um importante componente no controle da taxa e extensão de formação dos fungos micorrízicos, uma vez que características do sistema radicular, taxa de crescimento e alocação de carboidratos exercem forte influência na formação das associações micorrízicas (MANJUNATH \& HABTE , 1991; AMIJEE et al., 1993). A determinação de diferenças entre grupos de plantas ou indivíduos intimamente relacionados, na sua eficiência micorrízica, é um evento-chave na seleção de plantas hospedeiras com micorriza eficiente (LACKIE et al., 1988). A seleção de cultivares ou genótipos que são prontamente colonizados por fungos MA, pode ser um passo importante no sentido de se chegar a uma menor dependência de fertilizantes fosfatados (KRISHNA et al., 1985). O presente trabalho teve como objetivo determinar o coeficiente de determinação genotípica (b) do mamoeiro (Carica papaya L.) quanto à capacidade de se associar e responder ao fungo Gigaspora margarita.

\section{MATERIAL E MÉTODOS}

O experimento foi conduzido em condições de casa de vegetação nos meses de abril e maio de 1997, na Embrapa Mandioca e Fruticultura, utilizando-se de quatro genótipos representativos do grupo 'Solo' (06;12; 34 e 60), além da variedade 'Improved Sunrise Solo Line 72/12' (ISS 72/12) e quatro do grupo 'Formosa' ( $02 ; 07 ; 08$ e 23) além do híbrido 'Tainung $\mathrm{N}^{\mathrm{o}} 1$ '. Os dez genótipos foram submetidos à inoculação do fungo Gigaspora margarita, sendo um controle não inoculado, totalizando 20 tratamentos. O delineamento utilizado foi o de blocos casualizados, com quatro repetições, onde cada parcela foi representada por uma planta cultivada em um saco plástico preto com furos, contendo $1 \mathrm{dm}^{3}$ de solo.

Utilizou-se amostra de um Latossolo Amarelo álico textura franco argilo-arenosa (58 \% de areia, $12 \%$ de silte e $30 \%$ de argila), sob vegetação de pastagem nativa (gramíneas), coletado em área experimental da Embrapa Mandioca e Fruticultura, na profundidade de 10 a $30 \mathrm{~cm}$, apresentando as seguintes características químicas: $\mathrm{pH} 4,4 ; 3 \mathrm{mg} \mathrm{dm}^{-3} \mathrm{de} \mathrm{P} ; 31 \mathrm{mg} \mathrm{dm}^{-3} \mathrm{de} \mathrm{K}$; $8 \mathrm{mmol}_{\mathrm{c}}^{(+)} \mathrm{dm}^{-3} \mathrm{de} \mathrm{Ca} ; 4 \mathrm{mmol}_{\mathrm{c}}^{(+)} \mathrm{dm}^{-3} \mathrm{de} \mathrm{Mg} ; 8 \mathrm{mmol}_{\mathrm{c}}^{(+)} \mathrm{dm}^{-3} \mathrm{de} \mathrm{Al}$ e $1,61 \mathrm{~g} \cdot \mathrm{kg}^{-1}$ de matéria orgânica. $\mathrm{O}$ solo foi desinfestado com brometo de metila na dosagem de $393 \mathrm{~cm}^{3}$ do produto comercial
(Bromex) por $\mathrm{m}^{3}$ de solo e ventilado por 72 horas. A correção química do solo foi realizada aplicando-se uma dose equivalente a 1,6 t/ha de calcário na forma de uma mistura de $\mathrm{CaCO}_{3}+\mathrm{MgCO}_{3}$, P.A. (proporção de 4:1 $\mathrm{em} \mathrm{mmol}_{\mathrm{c}}^{(+)} \mathrm{de} \mathrm{Ca}: \mathrm{Mg}$ ). O fósforo foi aplicado na dose de $20 \mathrm{mg} \mathrm{dm}^{-3}$ de solo, forma de superfosfato triplo. Efetuou-se uma aplicação básica de nitrogênio $(20 \mathrm{mg} \mathrm{dm}-$ ${ }^{3}$ ), potássio $\left(80 \mathrm{mg} \mathrm{dm}^{-3}\right)$ e enxofre $\left(28 \mathrm{mg} \mathrm{dm}^{-3}\right)$, utilizando-se de sulfato de amônio e cloreto de potássio como fontes.

As sementes, obtidas de mamoeiros existentes no Banco de Germoplasma da Embrapa Mandioca e Fruticultura (BAGMamão), foram germinadas em mistura de areia + vermiculita (2:1), estando prontas para repicagem quando apresentavam um par de folhas definitivas.

A inoculação foi realizada com solo-inóculo depositado em orifício central feito no recipiente de cultivo. A seguir, efetuouse a repicagem da muda de mamoeiro de forma que as raízes ficaram em contato íntimo com o inóculo. O fungo G. margarita foi obtido junto à Empresa Maguari, localizada no município de Araguari-MG.

Micronutrientes foram aplicados, em cobertura, via solução nutritiva, em dose única, dez dias após a repicagem,

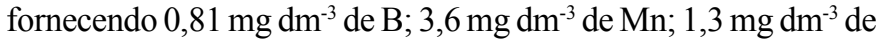

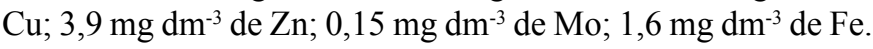
Adubação complementar de nitrogênio e enxofre foi realizada 25 dias após a repicagem, fornecendo $10 \mathrm{mg} \mathrm{dm}^{-3} \mathrm{de} \mathrm{Ne} 12,3 \mathrm{mg} \mathrm{dm}^{-3}$ de $\mathrm{S}$, via solução nutritiva. Aos 41 dias de cultivo (após a repicagem), coletou-se a parte aérea para a obtenção do peso de matéria seca e avaliação nutricional. Do sistema radicular, foram coletadas todas as raízes para avaliação do comprimento, utilizando-se do método da placa reticulada (NEWMAN, 1966). Posteriormente, fez-se uma amostragem de raízes para coloração e avaliação da colonização micorrízica total (AMBLER \& YOUNG, 1977).

Com os dados de produção de parte aérea, calculou-se a eficiência micorrízica de acordo com a fórmula:

(parte aérea de planta inoculada - parte aérea de planta não inoculada)

$\mathrm{EM}=\stackrel{\text { (parte aérea de planta inoculada) }}{\longrightarrow} \times 100$

Foi feita análise de variância para todos os caracteres individualmente, utilizando-se dos valores de quadrado médio de tratamento e do erro experimental para estimar o coeficiente de determinação genotípica (b) que mede a proporção da variância fenotípica total, que é de natureza genética, dado pela fórmula: $\mathrm{b}=\frac{\mathrm{VT}}{\mathrm{QT} / \mathrm{r}}$, onde:

$\mathrm{VT}=$ Componente quadrático da variação entre genótipos, dado por: $\mathrm{QT}-\mathrm{QR} / \mathrm{r}$

$\mathrm{QT}=$ Quadrado médio de tratamentos

$\mathrm{QR}=$ Quadrado médio do erro experimental

$r=$ número de repetições

\section{RESULTADOS E DISCUSSÃO}

Todos os genótipos dos dois grupos avaliados responderam significativamente à inoculação com relação à produção de parte aérea (Tabela 1). Entre eles, a biomassa diferiu apenas no grupo 'Formosa', na ausência de inoculação, 
demonstrando potencial genético diferenciado para produção de parte aérea. A inoculação também aumentou significativamente a altura de plantas e o número de folhas para a maioria dos genótipos.

Os genótipos do grupo 'Solo' apresentaram maior comprimento de raiz e menor relação parte aérea/comprimento de raízes (Tabela 1 e Figura 1), confirmando os dados de TRINDADE (1998). O comprimento de raízes é uma das características da planta que tem influência na resposta à inoculação de FMA (KOIDE, 1991), alterando a dependência da planta aos fungos. O grupo Solo apresentou uma variação de eficiência micorrízica de $10,5 \%$, com três genótipos apresentando valores na faixa de 60 \% (Figura 2); no grupo Formosa, a variação foi de 41,8\%, com a maioria dos valores na faixa de $70 \%$, indicando maior potencial de resposta desse grupo à inoculação. Neste grupo, a eficiência micorrízica do acesso 7 foi significativamente superior à observada para o acesso 2. No grupo 'Solo', os genótipos não diferiram quanto à eficiência micorrízica. Esta diferença intraespecífica foi constatada em citros (ONKARAYA et al., 1993), morango (KHANIZADEH et al., 1995) e banana (DECLERCK et al., 1995), entre outras culturas, embora em alfafa não tenha sido observada (O’BANNON et al., 1980). Isto indica a complexidade do sistema e mostra que há abertura para a inclusão da micorriza como um item de um programa de melhoramento, baseado na maximização da eficiência de absorção de nutrientes e uso pelas culturas que mostram variabilidade genotípica.

Para o grupo 'Solo’ não houve correlação significativa entre eficiência micorrízica e comprimento de raízes (plantas não inoculadas). Contudo, no grupo 'Formosa', houve uma correlação de $r=-0,94^{* *}$, demonstrando ser este caráter um ponto crítico para as plantas, tornando-as mais dependentes da micorriza. No grupo 'Formosa', o híbrido Tainung $\mathrm{N}^{\mathrm{o}} 1$ apresentou o mais baixo valor de comprimento radicular (Tabela 1), respondendo à inoculação neste caráter. De acordo com hipótese de TRINDADE (1998), o efeito de inoculação nesta variedade dá-se principalmente pelo efeito indireto de aumento do comprimento

TABELA 1 - Dados de crescimento de plantas de genótipos e variedades de mamoeiro dos grupos 'Solo' e 'Formosa', em função da inoculação de $G$. margarita. (NI=não inoculado; $\mathrm{I}=$ inoculado). ${ }^{1}$

\begin{tabular}{|c|c|c|c|c|c|c|c|c|c|c|c|c|}
\hline \multirow{3}{*}{$\frac{\text { G rupo/G enótipo }}{\text { S O L O }}$} & \multicolumn{3}{|c|}{$\begin{array}{c}\text { Parte aérea } \\
(\mathrm{g} / \mathrm{p} \text { lanta })\end{array}$} & \multicolumn{4}{|c|}{$\begin{array}{l}\text { A ltura de planta } \\
\text { (cm/planta) }\end{array}$} & \multicolumn{3}{|c|}{$\begin{array}{l}\text { Núm ero de } \\
\text { folhas }\end{array}$} & \multicolumn{2}{|c|}{$\begin{array}{c}\text { Comprimento de raiz } \\
(\mathrm{m} / \mathrm{pla} \text { la })\end{array}$} \\
\hline & N I & \multicolumn{2}{|l|}{ I } & \multicolumn{2}{|c|}{ N I } & \multicolumn{2}{|c|}{ I } & \multicolumn{2}{|c|}{ N I } & I & $\mathrm{N} \mathrm{I}$ & I \\
\hline & $0,10 \mathrm{~A} \mathrm{~b}$ & 0,34 & $\mathrm{~A} \mathrm{a}$ & 8,0 & $\mathrm{~A} \mathrm{~b}$ & 12,0 & $\mathrm{~A} \mathrm{a}$ & 5,5 & $\mathrm{~A} \mathrm{~b} \mathrm{~b}$ & $9,7 \mathrm{~A} \mathrm{a}$ & $2,67 \mathrm{~A} \mathrm{~b}$ & $5,30 \quad \mathrm{~A} \mathrm{a}$ \\
\hline 12 & $0,13 \mathrm{~A} \mathrm{~b}$ & 0,35 & $\mathrm{~A} \mathrm{a}$ & 7,6 & $\mathrm{~A} \mathrm{~b}$ & 10,9 & $\mathrm{~A}$ a & 6,8 & $\mathrm{~A} \mathrm{~B} \mathrm{a}$ & $8,3 \mathrm{~A} \mathrm{a}$ & $1,98 \mathrm{~A} \mathrm{a}$ & 2,58 \\
\hline 34 & $0,13 \mathrm{~A} \mathrm{~b}$ & 0,39 & $\mathrm{~A} \mathrm{a}$ & 8,4 & $\mathrm{~A} \mathrm{~b}$ & 11,6 & $\mathrm{~A} \mathrm{a}$ & 6,5 & $\mathrm{~A} \mathrm{~B} \mathrm{~b}$ & $8,7 \mathrm{~A} \mathrm{a}$ & $3,19 \mathrm{~A} \mathrm{a}$ & 4,36 \\
\hline 60 & $0,13 \mathrm{~A} \mathrm{~b}$ & 0,33 & $\mathrm{~A} \mathrm{a}$ & 7,4 & $\mathrm{~A} \mathrm{~b}$ & 10,3 & A B a & 5,0 & B b & $8,5 \mathrm{~A} \mathrm{a}$ & $1,55 \mathrm{~A} \mathrm{a}$ & $3,51 \mathrm{~A} \mathrm{~B}$ a \\
\hline $72 / 12$ & $0,12 \mathrm{~A} \mathrm{~b}$ & 0,40 & $\mathrm{~A} \mathrm{a}$ & 7,6 & $\mathrm{~A} \mathrm{~b}$ & 9,6 & $\mathrm{~A} \mathrm{~B} \mathrm{a}$ & 7,7 & $\mathrm{~A} \mathrm{a}$ & $8,3 \mathrm{~A} \mathrm{a}$ & $2,10 \mathrm{~A} \mathrm{a}$ & $3,80 \quad \mathrm{~A} \mathrm{~B}$ a \\
\hline m édia & 0,12 & 0,36 & & 7,8 & & 10,9 & & 6,3 & & 8,7 & 2,30 & 3,91 \\
\hline \multicolumn{13}{|l|}{ F O R M O S A } \\
\hline 02 & 0,20 & 0,32 & $\mathrm{~A} \mathrm{a}$ & 9,6 & $\mathrm{~A} \mathrm{~b}$ & 11,4 & $\mathrm{~A} \mathrm{a}$ & 7,3 & $\mathrm{~A}$ a & $7,3 \mathrm{~A} \mathrm{a}$ & $3,18 \mathrm{~A} \mathrm{a}$ & $3,74 \mathrm{~A} \mathrm{a}$ \\
\hline 07 & $0,06 \quad \mathrm{~B} \mathrm{~b}$ & 0,29 & $\mathrm{~A} \mathrm{a}$ & 5,3 & $\mathrm{C} \mathrm{b}$ & 8,2 & $\mathrm{~A}$ a & 4,3 & $\mathrm{~B} \mathrm{~b}$ & $7,5 \mathrm{~A} \mathrm{a}$ & $1,13 \mathrm{~A} \mathrm{~b}$ & $3,76 \mathrm{~A} \mathrm{a}$ \\
\hline 08 & 0,11 A B b & 0,31 & $\mathrm{~A} \mathrm{a}$ & 7,3 & $\mathrm{~B} \mathrm{C} \mathrm{b}$ & 8,9 & $\mathrm{~A} \mathrm{a}$ & 5,5 & $\mathrm{~A} \mathrm{~B} \mathrm{~b}$ & $8,3 \mathrm{~A} \mathrm{a}$ & $1,14 \mathrm{~A} \mathrm{~b}$ & $3,91 \mathrm{~A} \mathrm{a}$ \\
\hline 23 & $0,08 \quad \mathrm{~B} \mathrm{~b}$ & 0,28 & $\mathrm{~A} \mathrm{a}$ & 6,4 & $\mathrm{~B} \mathrm{C} \mathrm{b}$ & 8,0 & $\mathrm{~A} \mathrm{a}$ & 4,5 & $\mathrm{~B}$ a & $6,0 \mathrm{~A} \mathrm{a}$ & $0,90 \mathrm{~B} \mathrm{a}$ & $1,36 \mathrm{~A} \mathrm{a}$ \\
\hline $\mathrm{T}$ ainung $\mathrm{N}^{\circ} 1$ & 0,07 & 0,25 & $\mathrm{~A} \mathrm{a}$ & 7,7 & $\mathrm{~A} \mathrm{~B} \mathrm{a}$ & 9,1 & $\mathrm{~A} \mathrm{a}$ & 5,0 & $\mathrm{~A} \mathrm{~B} \mathrm{~b}$ & $7,3 \mathrm{~A} \mathrm{a}$ & $0,80 \mathrm{~B} \mathrm{~b}$ & $1,79 \mathrm{~A} \mathrm{a}$ \\
\hline m é dia & 0,10 & 0,29 & & 7,3 & & 9,1 & & 5,3 & & 7,3 & 1,43 & 2,91 \\
\hline
\end{tabular}

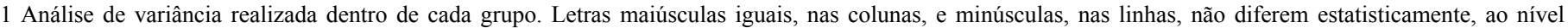
de $5 \%$ de probabilidade, pelo teste de Tukey.

TABELA 2 - Teores de nutrientes e conteúdo de fósforo na parte aérea de plantas de genótipos e variedades de mamoeirodos grupos 'Solo’ e ‘Formosa', em função da inoculação de G. margarita. (NI=não inoculado; I=inoculado).

\begin{tabular}{|c|c|c|c|c|c|c|c|c|c|c|}
\hline $\begin{array}{l}\text { G R UPO/ } \\
\text { Genótipo }\end{array}$ & \multicolumn{2}{|c|}{$\begin{array}{l}\text { Fós foro } \\
\left(\mathrm{g} \mathrm{kg}^{-1}\right)\end{array}$} & \multicolumn{2}{|c|}{$\begin{array}{c}\text { Fós foro } \\
(\mu \mathrm{g} / \mathrm{planta})\end{array}$} & \multicolumn{2}{|c|}{$\begin{array}{c}\text { Potássio } \\
\left(\mathrm{g} \mathrm{kg}^{-1}\right)\end{array}$} & \multicolumn{2}{|c|}{$\begin{array}{c}\text { Cobre } \\
\left(\mu \mathrm{g} \mathrm{g}^{-1}\right)\end{array}$} & \multicolumn{2}{|c|}{$\begin{array}{c}Z \text { Zinco } \\
\left(\mu \mathrm{g} \mathrm{g}^{-1}\right)\end{array}$} \\
\hline 06 & 0,12 & 0,28 & 120 & 952 & 1,35 & 3,45 & 8,5 & 12,6 & 33,8 & 40,0 \\
\hline 60 & 0,10 & 0,23 & 130 & 759 & 2,40 & 2,79 & 9,4 & 9,7 & 33,4 & 32,3 \\
\hline IS S $72 / 12$ & 0,08 & 0,25 & 96 & 1000 & 1,30 & 2,92 & 7,2 & 13,8 & 24,4 & 36,3 \\
\hline média & 0,12 & 0,25 & 142 & 897 & 1,96 & 3,20 & 9,7 & 12,3 & 36,5 & 35,8 \\
\hline 07 & 0,09 & 0,22 & 54 & 638 & 1,06 & 2,53 & 7,3 & 9,7 & 28,7 & 32,3 \\
\hline 08 & 0,08 & 0,19 & 88 & 589 & 1,61 & 2,71 & 7,1 & 7,8 & 40,8 & 28,4 \\
\hline 23 & 0,10 & 0,30 & 80 & 840 & 0,78 & 2,66 & 8,3 & 12,7 & 28,8 & 36,5 \\
\hline Tainung $\mathrm{N}^{\mathrm{O}} 1$ & 0,06 & 0,29 & 42 & 725 & 0,92 & 2,71 & 7,7 & 12,5 & 19,6 & 49,5 \\
\hline média & 0,10 & 0,25 & 125 & 718 & 1,38 & 2,65 & 8,0 & 10,6 & 32,6 & 34,8 \\
\hline
\end{tabular}

TABELA 3 - Valores do coeficiente de determinação genotípico, "b", em percentagem, para diferentes caracteres de crescimento das plantas e sua relação com o fungo MA.

\begin{tabular}{cccccc}
\hline \multirow{2}{*}{ Grupo } & \multicolumn{5}{c}{ Caracteres } \\
\cline { 2 - 6 } & Parte aérea & Comprimento de raiz & Altura de planta & Eficiência micorrízica & Colonização \\
\hline Solo & 48,7 & 1,2 & $<0$ & 36,7 & 85,3 \\
Formosa & 89,6 & 81,5 & 88,0 & 81,5 & 0 \\
\hline
\end{tabular}



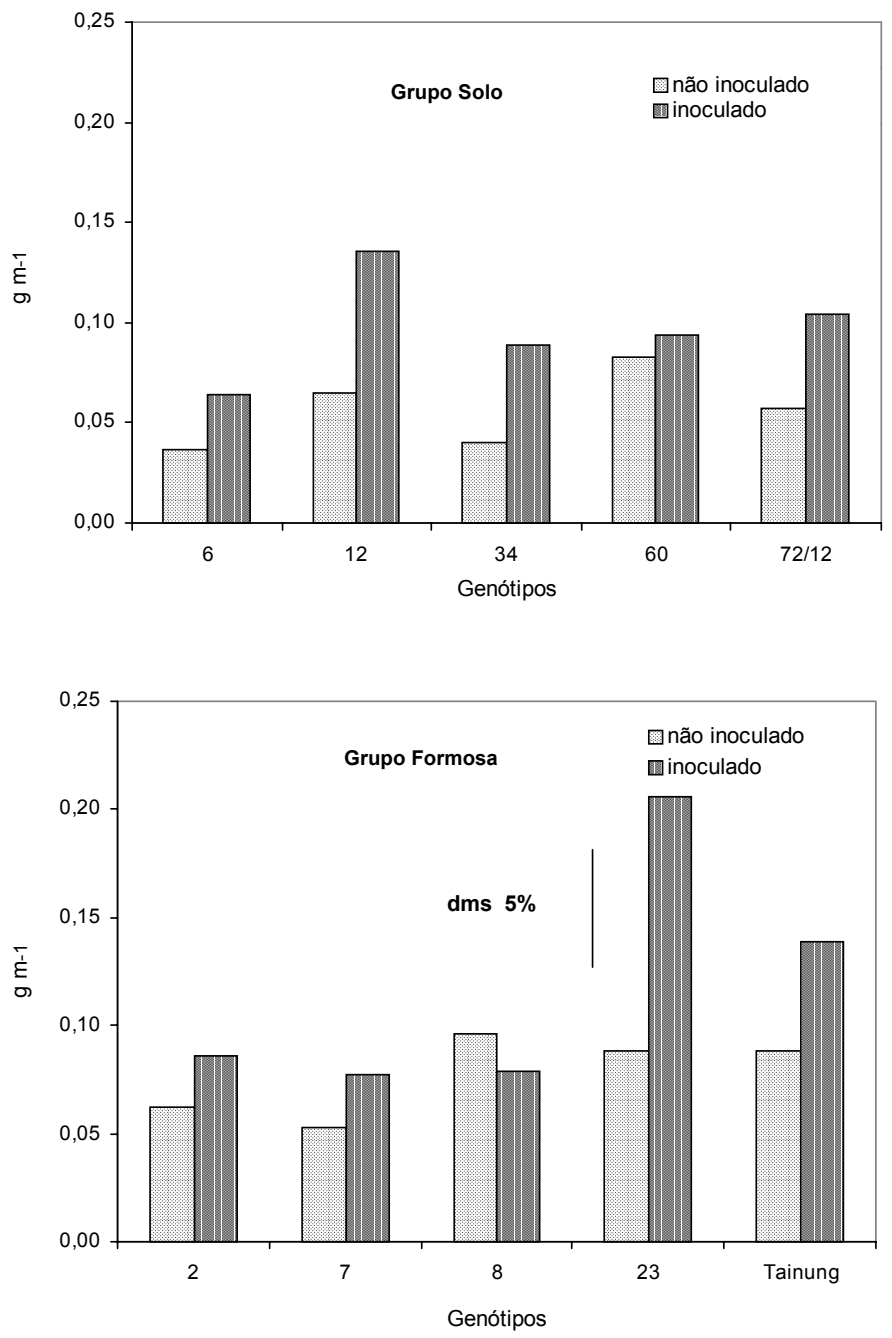

FIGURA 1 - Relação parte aérea/comprimento de raízes de plantas de genótipos de mamão pertencentes aos dois grupos, inoculadas ou não com G. margarita.

radicular. Além de Tainung $\mathrm{N}^{\mathrm{o}} 1$, os genótipos 07 e 08 do grupo Formosa também responderam à inoculação, enquanto no grupo Solo, apenas um genótipo (06) teve o comprimento radicular significativamente aumentado com a inoculação. Não é possível afirmar se a característica de menor ou maior produção de raízes evoluiu com a formação da micorriza ou se esta se expressou a partir da característica de produção do sistema radicular pela planta. Entretanto, sendo este o cenário real da relação fungoplanta de um genótipo vegetal e, sendo a associação micorrízica uma regra na natureza, a micorriza deve compensar pela menor produção de raiz, sendo energeticamente mais eficiente, como sugerido por JOHNSON et al. (1997). Além do mais, a eliminação da colonização micorrízica, seja por manejo inapropriado da adubação, seja pelo melhoramento, vai eliminar os efeitos benéficos na estrutura do solo, relações hídricas e tolerância a doenças, além da nutrição da planta (SMITH et al., 1993).

Dentre os nutrientes avaliados, a inoculação aumentou a concentração de fósforo em ambos os grupos, em todos os genótipos. Proporcionalmente, foi o nutriente mais influenciado pela inoculação. As plantas do grupo Solo apresentaram tendência de maior acúmulo de fósforo (Tabela 2). Entretanto, o aumento no teor e conteúdo de fósforo com a inoculação foi

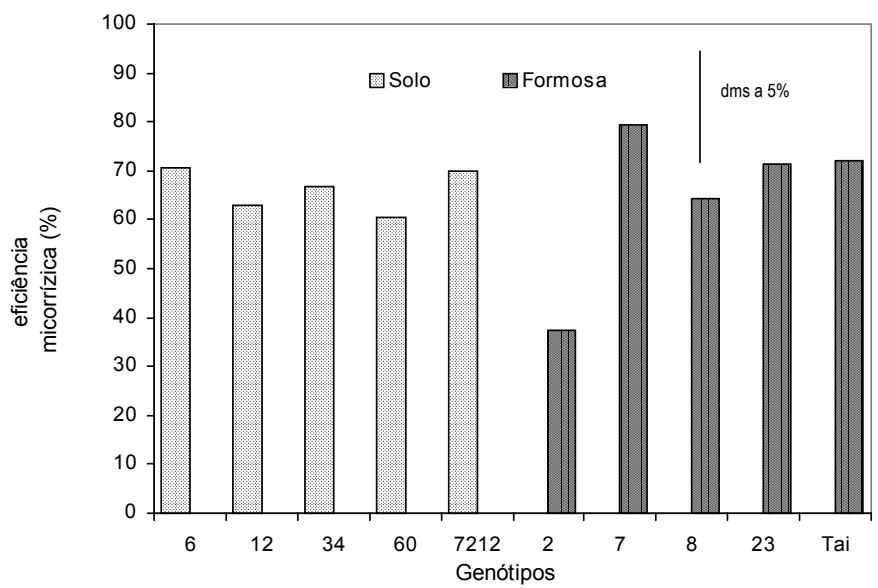

FIGURA 2 - Eficiência micorrízica de plantas de genótipos de mamão pertencentes aos dois grupos, inoculadas ou não com G. margarita.

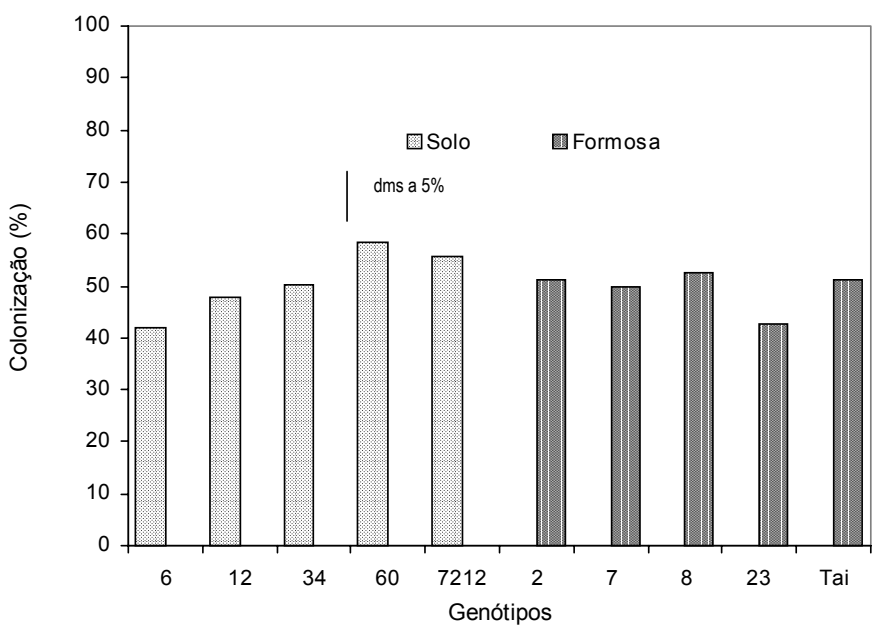

FIGURA 3 - Percentual de colonização micorrízica de plantas de genótipos de mamão pertencentes aos dois grupos, inoculadas ou não com G. margarita.

proporcionalmente maior nos genótipos do grupo 'Formosa', indicando que as plantas desse grupo, não inoculadas, apresentam um maior déficit e que a inoculação apresenta maior efeito na redução desse déficit. Ou seja, o efeito da colonização no aumento do suprimento de $\mathrm{P}$ é proporcionalmente maior nas plantas do grupo 'Formosa' . A correlação entre o fósforo absorvido pelas plantas não inoculadas e a eficiência micorrízica foi negativa e significativa para o grupo Formosa $\left(r=-0,97^{* *}\right)$. Este dado, juntamente com a correlação mostrada entre eficiência micorrízica e comprimento radicular, comprovam que plantas com menor capacidade de suprimento apresentam maior eficiência da colonização micorrízica. Genótipos que são mais dependentes da micorriza para um adequado suprimento de nutrientes, provavelmente, apresentam baixa capacidade de exploração do sistema radicular (BOLAN et al., 1987; MANJUNATH \& HABTE, 1991).

A inoculação promoveu maior absorção de $\mathrm{K} \mathrm{em}$ todos os genótipos dos dois grupos. Considerando-se que o $\mathrm{K}$ é um elemento de elevada mobilidade no solo e que foi fornecido em teores considerados suficientes para a cultura, os dados da Tabela 2 indicam uma baixa capacidade, intrínseca, de absorção de nutrientes pelas raízes da espécie Carica papaya. Em alguns 
genótipos, a absorção de $\mathrm{K}$ atingiu valores acima de 100\% em relação às plantas não colonizadas. Para o $\mathrm{Cu}$, plantas inoculadas, nos dois grupos, apresentaram maior concentração. No caso do $\mathrm{Zn}$, houve grande variação entre os genótipos de um mesmo grupo com relação ao padrão de concentração em função da inoculação.

Dentro dos dois grupos de mamoeiros avaliados, a variedade 'ISS 72/12' e o híbrido Tainung No 1 apresentaram menor capacidade de absorção dos nutrientes. Os efeitos da inoculação sobre a absorção de nutrientes foram proporcionalmente maiores para estes genótipos, dentro de cada grupo. Pelo fato de esses genótipos terem sido selecionados para ambientes otimizados, é provável que os genótipos comerciais apresentem características mais apropriadas a estas condições. Em solo com baixo teor de P, como é o caso, estas plantas podem sofrer redução na capacidade de absorção do nutriente.

A colonização micorrízica atingiu valores entre $40 \%$ e $60 \%$ (Figura 3), não havendo diferenças significativas entre os genótipos do grupo 'Formosa'. Dentro do grupo 'Solo', o genótipo 06 apresentou o menor valor, mas não houve correlação significativa com a eficiência micorrízica.

Os valores calculados do coeficiente de determinação genotípico (b) indicam grandes diferenças entre os genótipos dos dois grupos de plantas (Tabela 3). Para o grupo 'Solo', o valor 'b' foi mais elevado para colonização micorrízica, indicando que este carácter apresenta grande probabilidade de ser modificado por melhoramento genético. LACKIE et al. (1988) encontraram resultado semelhante em famílias de meio-irmãos de alfafa. Entretanto, caracteres como comprimento de raiz e altura de plantas, de acordo com o experimento, mostraram-se bastante estáveis, difíceis de serem modificados geneticamente. A eficiência micorrízica apresentou valor próximo a 40\%, e que está, portanto, dissociado das variações ocorridas no sistema radicular, como já demonstrado para esse grupo. O valor ' $b$ ' é uma propriedade não somente de um carácter, mas também da população e das circunstâncias de ambiente a que os indivíduos estão sujeitos (FALCONER, 1987). Nas condições empregadas, os genótipos do grupo 'Formosa' apresentam maiores probabilidades de serem alterados mediante programas de melhoramento, considerando-se o acúmulo de parte aérea, comprimento de raízes e altura de plantas, todos com coeficientes acima de $80 \%$. Entretanto, diferentemente destes caracteres e dos dados verificados para o grupo Solo, a taxa de colonização não pode sofrer grandes modificações, segundo o valor " $b$ " calculado. Corroborando a ausência de correlação entre taxa de colonização e eficiência micorrízica, este caráter, para esse grupo, apresentou coeficiente de determinação genotípico acima de $80 \%$, sugerindo que trabalhos de melhoramento, tendo o comprimento de raiz como um dos indicadores, podem gerar genótipos com diferentes graus de dependência aos fungos MA, em função da correlação negativa entre comprimento de raiz e eficiência micorrízica.

\section{CONCLUSÕES}

1. Os genótipos dos grupos Solo e Formosa responderam à inoculação do fungo MA.
2. O grupo Formosa apresentou menor comprimento de raiz e maior demanda na forma da proporção entre parte aérea e comprimento de raiz.

3. A eficiência micorrízica e a colonização radicular média para os dois grupos de plantas foram similares, situando-se em $60 \% \mathrm{e}$ $50 \%$, respectivamente.

4. Não houve correlação, dentro de um mesmo grupo, entre taxa de colonização e eficiência micorrízica.

5. Nos dois grupos, a absorção de $\mathrm{P}, \mathrm{K}$ e $\mathrm{Cu}$ foi aumentada pela inoculação.

6. O coeficiente ' $b$ ' demonstrou que o melhoramento de plantas pode modificar a produção de parte aérea, comprimento de raízes, altura de plantas e a eficiência micorrízica do grupo Formosa; para o grupo Solo, este efeito mostrou-se mais provável para colonização radicular, produção de parte aérea e menos provável para eficiência micorrízica, comprimento de raiz e altura de planta.

\section{REFERÊNCIA BIBLIOGRÁFICAS}

AMBLER, J.R.; YOUNG, J.L. Techniques for determining root length infected by vesicular-arbuscular mycorrhizae. Soil Science Society of American Journal, Madison, v. 4, n. 3, p.551-556, 1977.

AMIJEE, F.; STRIBLEY, D.P.; TINKER, P B. The development of endomycorhizal root systems: effects of soil phosphorus and fungal colonization on the concentration of soluble carbohydrates in roots. The New Phytologist, Oxford, v. 123, n. 2, p.297-306,1993.

BOLAN, N. S. A critical review on the role of mycorrhizal fungi in the uptake of phosphorus by plants. Plant and Soil, The Hague, v. 134, n. 2,p.189-207, 1987.

DECLERCK, S.; PLENTCHETTE, C.; STRULLU,D.G. Mycorrhizal dependency of banana (Musa acuminata, AAA group) cultivar. Plant and Soil, Dodrecht, v. 176, n. 2, p.183-187, 1995.

FALCONER, D.S. Introdução à genética quantitativa. Viçosa,M.G., UFV. Imp. Univ., 1987. 279p.

FAO. Disponível: Site FAO (05 Abril, 2000). URL: http:// www.fao.org.. Consultado em 28 junho, 2000

GERDEMANN, J. W. Vesicular-arbuscular mycorrhizae. In: TORREY, J.G.; CLARKSON, D.T.(Ed), The development and function of roots. London: Academic Press, 1975. p. 575-91.

GIACOMETTI, D.C. Papaya breeding. Acta Horticulturae, Wageningen, n.196, p.53-60, 1987

JOHNSON, N.C.; GRAHAM, J.H.; SMITH, F. A. Functioning of mycorrhizal associations along the mutualism-parasitism continum. The New Phytologist, Oxford, v. 135, n. 4, p.575-585, 1997.

KHANIZADEH, S.; HAMEL, C.; KIANMEHR, H.; BUSZARD, D.; SMITH, D.L. Effects of three vesicular-arbuscular mycorrhizae species and phosphorus on reproductive and vegetative growth 
of three strawberry cultivars. Journal of Plant Nutrition, Monticello, v. 18, n. 6, p.1073-1079, 1995.

KOIDE, J.T. Nutrient supply, nutrient demand and plant response to mycorrhizal infection. The New Phytologist, Oxford, v. 117, n.3, p.365-386, 1991.

KRISHNA, K.R.; SHETTY, K.G.; DART,P.J.; ANDREWS, D.J. Genotype dependent variation in mycorrhizal colonization and response to inoculation of pearl millet. Plant and Soil, Dodrecht, v. 86, n. 1, p.113-125, 1985.

LACKIE, S.M.; BOWLEY, S.R.; PETERSON, R.L. Comparison of colonization among half-sib families of Medicago sativa L. by Glomus versiforme (Daniels and Trappe) Berch. The New Phytologist, Oxford, v. 108, n. 4, p.477-482, 1988.

MANJUNATH, A.; HABTE, M. Root morphological characteristics of host species having distinct mycorrhizal dependence. Canadian Journal of Botany, Otawa, v. 69, n.5, p.671$676,1991$.

MOSSE, B. Advances in the study of vesicular-arbuscular mycorrhiza. Annual Review of Phytopathology, Palo Alto, v. 11, n. 1, p.171-96, 1973.
NEWMAN, E. I. A method of estimating the total length of root in a sample. Journal of Applied Ecology, Oxford, v. 3, n.2, p.139$145,1966$.

O‘BANNON, J. H.; EVANS, D. W.; PEADEN, R. N. Alfafa varietal response to seven isolates of vesicular-arbuscular mycorrhizal fungi. Canadian Journal of Plant Science, Otawa, v. 60, n.3, p. 859-863, 1980.

ONKARAYA, H.; MOHANDAS, S. Studies on dependency of citrus rootstocks to VAM inoculation in Alfisol soil. Advances in Horticulture and Forestry, Hessaraghatta, v. 3, n.1, p.81-91, 1993.

SMITH, S.E.; ROBSON, A D.; ABBOTT, L.K. The involvement of mycorrhizas in assessment of genetically dependent efficiency of nutrient uptake and use. In: RANDALL et al. (Ed) Genetic aspects of plant mineral nutrition. Dodrecht: Kluwer Academic Publishers, 1993. p. 221-231.

TRINDADE, A .V. Fungos micorrízicos arbusculares em mamoeiro. 1998.117f Tese (Doutorado) - Universidade Federal de Lavras, Lavras, 1998. 